\title{
NAVIGATION ACCURACY GUIDELINES FOR ORBITAL FORMATION FLYING
}

\author{
J. Russell Carpenter* \\ NASA Goddard Space Flight Center, Greenbelt, MD 20771 \\ and Kyle T. Alfriend ${ }^{\dagger}$ \\ Texas A\&M University, College Station, TX 77843
}

\begin{abstract}
Some simple guidelines based on the accuracy in determining a satellite formation's semi-major axis differences are useful in making preliminary assessments of the navigation accuracy needed to support such missions. These guidelines are valid for any elliptical orbit, regardless of eccentricity. Although maneuvers required for formation establishment, reconfiguration, and station-keeping require accurate prediction of the state estimate to the maneuver time, and hence are directly affected by errors in all the orbital elements, experience has shown that determination of orbit plane orientation and orbit shape to acceptable levels is less challenging than the determination of orbital period or semi-major axis. Furthermore, any differences among the member's semi-major axes are undesirable for a satellite formation, since it will lead to differential along-track drift due to period differences. Since inevitable navigation errors prevent these differences from ever being zero, one may use the guidelines this paper presents to determine how much drift will result from a given relative navigation accuracy, or conversely what navigation accuracy is required to limit drift to a given rate. Since the guidelines do not account for non-two-body perturbations, they may be viewed as useful preliminary design tools, rather than as the basis for mission navigation requirements, which should be based on detailed analysis of the mission configuration, including all relevant sources of uncertainty.
\end{abstract}

\section{Introduction}

One of the most significant differences between many formation flying satellite missions that are currently of interest, and the intentionally close approaches that past missions have performed e.g. rendezvous, docking, and proximity operations of the Space Shuttle - is the need for long-term and efficient maintenance of relatively close formations. As of 2002, separations of a few hundred

\footnotetext{
*Aerospace Engineer, Flight Dynamics Analysis Branch, Mission Engineering and Systems Analysis Division. Senior Member, AIAA.

†Wisenbaker II Professor, Aerospace Engineering Dept. Fellow, AIAA.
} 
kilometers have been achieved for long-duration formations, and in the next few years separations in the range of $100 \mathrm{~m}$ to $10 \mathrm{~km}$ are expected to be attempted. Ref. 1 describes some relevant aspects of one such recent mission.

Many perturbations affect the accuracy of maneuvers required to maintain these formations, but in principle the maneuver planning process can accommodate all known perturbations. However, the navigation errors at the time of the maneuver computation will form a lower bound on the accuracy of the maneuvers, since even a perfectly executed maneuver will "lock in" the navigation errors. Indeed, Ref. 2 suggests that velocity uncertainty may be the limiting technology for formation flying.

Although the errors in all the states will affect the maneuver, as Ref. 3 discusses, semi-major axis error is the most difficult to estimate. An essential point of Ref. 3 is that (for circular orbits) semi-major axis uncertainty depends on three quantities: the radial position error, the along-track velocity error, and the correlation between these errors, which arises due to the the conservation of energy. The most notable consequence of semi-major axis differences for a formation of satellites is relative drift in the direction of the orbital path, which Figure 2 illustrates. For the relatively high-thrust, short burning propulsion systems flown on current missions, the result of this drift will be more frequent stationkeeping maneuvers. For "continuous" low-thrust systems proposed for many upcoming missions, this drift will be a random error that must be counteracted, limited by the accuracy of the navigation feedback signal. Figure 1 illustrates the effect of the various contributors to along-track drift in a circular orbit, based on the relationships of Ref. 3 . To interpret Figure 1, consider a navigation system that can produce radial accuracy of $10 \mathrm{~cm}$, and speed accuracy of $0.1 \mathrm{~mm} / \mathrm{sec}$. Figure 1 indicates that the corresponding along-track drift may be on the order of $1 \mathrm{~m} /$ orbit, and could be as poor as about $3 \mathrm{~m} /$ orbit, if the correlation between radius and speed, $\rho_{r v}$, is poor.

Ref. 4 describes a necessary condition for obtaining a no-drift solution in eccentric orbits in a deterministic setting. Ref. 1 describes requirements on relative semi-major axis knowledge and control for relative drift of the GRACE mission due to differential drag. The contribution of the development below is to describe and quantify the relative drift issue in terms of the (nondeterministic) navigation errors for elliptical orbits, generalizing the results of Ref. 3 . The paper concludes with examples in low circular and high apogee elliptical Earth orbit missions.

\section{Development}

For every complete revolution in any two-body elliptical orbit, an error in knowledge of the orbit period, $\delta T_{p}$, results in an along-track error growth, $\delta s$, proportional to the speed (velocity magnitude), $v$,

$$
\delta s\left(f_{i}+2 \pi\right)=-v\left(f_{i}+2 \pi\right) \delta T_{p}
$$


where $f_{i}$ is the initial true anomaly. It is not hard to show (viz. Ref. 3) that

$$
\delta T_{p}=3 \pi \sqrt{\frac{a}{\mu}} \delta a
$$

where $a$ is the semi-major axis and $\mu$ is the gravitational constant, $G M$, so that

$$
\delta s\left(f_{i}+2 \pi\right)=-3 \pi v\left(f_{i}+2 \pi\right) \sqrt{\frac{a}{\mu}} \delta a
$$

At periapse,

$$
v(0)=v(0,2 \pi, \ldots)=\sqrt{\frac{\mu}{a}} \sqrt{\frac{1+e}{1-e}}
$$

where $e$ is the eccentricity, and so the in-track growth per orbit at periapse is

$$
\delta s(2 \pi)=\delta s(2 \pi, 4 \pi, \ldots)=-3 \pi \sqrt{\frac{1+e}{1-e}} \delta a
$$

while at apoapse,

$$
v(\pi)=v(\pi, 3 \pi, \ldots)=\sqrt{\frac{\mu}{a}} \sqrt{\frac{1-e}{1+e}}
$$

and hence the in-track growth per orbit at apoapse is

$$
\delta s(3 \pi)=\delta s(3 \pi, 5 \pi, \ldots)=-3 \pi \sqrt{\frac{1-e}{1+e}} \delta a
$$

Since according to Eq. (5) or (7) the along-track error growth per orbit is linear in the semimajor axis error, their error distributions share this linear relationship. For example their standard deviations are related by

$$
\sigma_{s}(\pi)=3 \pi \sqrt{\frac{1-e}{1+e}} \sigma_{a}
$$

Ref. 3 gives the following relation, valid for circular orbits, among semi-major axis error, radius error, speed error, and the correlation between radius and speed errors, $\rho_{r v}$ :

$$
\sigma_{a}=2 \sqrt{\sigma_{r}^{2}+\frac{2}{n} \rho_{r v} \sigma_{r} \sigma_{v}+\frac{1}{n^{2}} \sigma_{v}^{2}}
$$

where $n$ is the mean motion. Note that the velocity terms in Eq. (9) are with respect to an inertial frame. Relative to a frame rotating at the (constant) orbital angular velocity, such as Hill's frame,

$$
\sigma_{a}=2 \sqrt{4 \sigma_{r}^{2}+\frac{4}{n} \rho_{r \dot{y}} \sigma_{r} \sigma_{\dot{y}}+\frac{1}{n^{2}} \sigma_{\dot{y}}^{2}}
$$


where $\dot{y}$ in Eq. (10) refers to the along-track component of velocity relative to the rotating frame.

The general form of the semi-major axis variance, also given in Ref. 3 , is

$$
\sigma_{a}^{2}=A\left(\mathbf{x}_{r e f}\right) P_{\mathbf{x}} A\left(\mathbf{x}_{r e f}\right)^{\mathrm{T}}
$$

where the state is $\mathbf{x}^{T}=\left[\mathbf{r}^{T}, \mathbf{v}^{T}\right], \mathbf{r}$ is the position, $\mathbf{v}$ is the velocity, $P_{\mathbf{x}}$ is the state error covariance, $\mathbf{x}_{\text {ref }}$ is given, and

$$
A(\mathbf{x})=\left.2 a^{2}\left[\frac{\mathbf{r}^{\mathrm{T}}}{r^{3}}, \frac{\mathbf{v}^{\mathrm{T}}}{\mu}\right]\right|_{\mathbf{x}_{\text {ref }}}
$$

Applying Eq. (11) at apoapse, and using Eq. (7), it is not hard to show that Eq. (9) may be generalized to

$$
\left.\frac{\sigma_{a}^{2}}{4}\right|_{f=\pi, 3 \pi, \ldots}=\frac{\sigma_{r}^{2}}{(1+e)^{4}}+\frac{2}{n} \sqrt{\frac{1-e}{(1+e)^{5}}} \rho_{r v} \sigma_{r} \sigma_{v}+\frac{1}{n^{2}}\left(\frac{1-e}{1+e}\right) \sigma_{v}^{2}
$$

or similarly at periapse to

$$
\left.\frac{\sigma_{a}^{2}}{4}\right|_{f=0,2 \pi, \ldots}=\frac{\sigma_{r}^{2}}{(1-e)^{4}}+\frac{2}{n} \sqrt{\frac{1+e}{(1-e)^{5}}} \rho_{r v} \sigma_{r} \sigma_{v}+\frac{1}{n^{2}}\left(\frac{1+e}{1-e}\right) \sigma_{v}^{2}
$$

To get an expression for the semi-major axis variance at any point in the orbit, the following relationships are useful:

$$
\begin{aligned}
v_{x} & =\sqrt{\frac{\mu}{p}} e \sin f \\
v_{y} & =\sqrt{\frac{\mu}{p}} \frac{p}{r} \\
\sqrt{\frac{\mu}{p}} & =\frac{1}{\eta} \sqrt{\frac{\mu}{a}} \\
& =\frac{a n}{\eta} \\
\frac{\mu}{r^{2}} & =\frac{\mu}{p^{2}}\left(\frac{p}{r}\right)^{2} \\
& =\frac{a n^{2}}{\eta^{4}}\left(\frac{p}{r}\right)^{2}
\end{aligned}
$$

where $\eta=\sqrt{1-e^{2}}$. From the definition of Eq. (12), $\delta a=A\left(\mathbf{x}_{r e f}\right) \delta \mathbf{x}$, which may be written in terms of radial and along-track components, $x$ and $y$, respectively, as

$$
\delta a=\frac{2 a^{2}}{\mu}\left[v_{x} \delta v_{x}+v_{y} \delta v_{y}+\frac{\mu}{r^{2}} \delta r\right]
$$


Using Eqs. (15) - (20) in Eq. (21), it is not hard to show that

$$
\delta a=2\left\{\frac{1}{n \eta}\left[e \sin (f) \delta v_{x}+(1+e \cos f) \delta v_{y}\right]+\frac{(1+e \cos f)^{2}}{\eta^{4}} \delta r\right\}
$$

Taking the expectation of the square of Eq. (22), and assuming a zero mean, results in the following expression for the semi-major axis variance at any point in an elliptical orbit:

$$
\begin{aligned}
\frac{\sigma_{a}^{2}}{4}=\frac{(1+e \cos f)^{4}}{\eta^{8}} \sigma_{r}^{2}+\frac{1}{(n \eta)^{2}}\left[(e \sin f)^{2} \sigma_{v_{x}}^{2}\right. & \left.+(1+e \cos f)^{2} \sigma_{v_{y}}^{2}\right] \\
+\frac{2(1+e \cos f)^{2}}{n \eta^{5}}\left[(e \sin f) \rho_{r v_{x}} \sigma_{r} \sigma_{v_{x}}+(1+e \cos f) \rho_{r v_{y}} \sigma_{r} \sigma_{v_{y}}\right] & +\frac{2 e(\sin f+e \sin f \cos f)}{(n \eta)^{2}} \rho_{v_{x} v_{y}} \sigma_{v_{x}} \sigma_{v_{y}}
\end{aligned}
$$

If the correlations between radius and radial velocity, $\rho_{r v_{x}}$, and between radial velocity and alongtrack velocity, $\rho_{v_{x} v_{y}}$, can be assumed to be insignificant, then the following simpler expression results:

$$
\begin{aligned}
\frac{\sigma_{a}^{2}}{4}=\frac{(1+e \cos f)^{4}}{\eta^{8}} \sigma_{r}^{2}+\frac{1}{(n \eta)^{2}}\left[(e \sin f)^{2} \sigma_{v_{x}}^{2}+(1+e \cos f)^{2} \sigma_{v_{y}}^{2}\right] & \\
& +\frac{2(1+e \cos f)^{3}}{n \eta^{5}} \rho_{r v_{y}} \sigma_{r} \sigma_{v_{y}}
\end{aligned}
$$

Figure 3 illustrates Eq. (24) for a particular example. Note that different relationships among $\sigma_{r}$, $\sigma_{v_{y}}$, and $\sigma_{v_{x}}$ than Figure 3 shows produce quite different results.

In most satellite formations, it is desirable for the satellites to minimize their semi-major axis differences so as to minimize relative along-track drift. The general form of the variance of the relative semi-major axis between any two satellties $i$ and $j$, also given in Ref. 3 , is

$$
\sigma_{\Delta a}^{2}=A\left(\mathbf{x}_{i}\right) P_{i} A\left(\mathbf{x}_{i}\right)^{\mathrm{T}}+A\left(\mathbf{x}_{j}\right) P_{j} A\left(\mathbf{x}_{j}\right)^{\mathrm{T}}-A\left(\mathbf{x}_{i}\right) P_{i j} A\left(\mathbf{x}_{j}\right)^{\mathrm{T}}-A\left(\mathbf{x}_{j}\right) P_{i j}^{\mathrm{T}} A\left(\mathbf{x}_{i}\right)^{\mathrm{T}}
$$

where $P_{i j}=P_{\mathbf{x}_{i} \mathbf{x}_{j}}$. To approximate and simplify Eq. (25), assume that the satellites in the formation have approximately the same $a$ and $e$, that their navigation errors have the same distributions, and that $P_{i j}=\rho_{i j} P_{i}$. Then, the version of Eqs. (13) $-(14)$ and (23) $-(24)$ corresponding to relative semi-major axis errors is

$$
\sigma_{\Delta a}=\sqrt{2-2 \rho_{i j}} \sigma_{a}
$$

Therefore, as in Eq. (8), the standard deviation of the in-track drift per orbit between any two satellites in a formation, evaluated at each apoapse, is related to their relative semi-major axis 
standard deviation by

$$
\sigma_{\Delta s}(3 \pi)=-3 \pi \sqrt{\frac{1-e}{1+e}} \sigma_{\Delta a}
$$

In a good orbital navigation filter, $-1<\rho_{r v} \leq-.9$, due to the dynamical constraint imposed on the estimates by conservation of energy*. A consequence of the high correlation between radius and speed errors is that speed error may be viewed as a dependent variable of radius error. Lear ${ }^{5}$ gives the following approximation of the speed error required to "balance" a corresponding radius error:

$$
\sigma_{v} \approx \frac{n}{\sqrt{1-e^{2}}} \frac{\sigma_{r}}{1+\frac{1}{4} e^{2}+\frac{1}{64} e^{4}+\frac{1}{256} e^{6}}
$$

Eq. (28) gives the speed error that produces the same size error in semi-major axis as the given radius error. Table 1 gives some numerical examples of Eq. (28). If Eq. (28) holds, then in-track drift per orbit may be viewed as a function of the radius error only.

If there are no relative measurements, but instead each satellite's absolute state is estimated separately, e.g. from GPS pseudoranges, $\rho_{i j}$ may be quite small. If the filter processes relative measurements such as cross-link ranges and/or GPS meausurement differences, $\rho_{i j} \approx 0.9$ may be a reasonable assumption. Based on the assumptions that $\rho_{i j}=0.9$, that the approximate velocity constraint of Eq. (28) is valid, and that the radius and speed are well-correlated $\left(\rho_{r v}=-.9\right)$, Figure 4(a) gives some examples of relative drift due to semi-major axis error for various eccentricities, for Earth-orbiting formations. The left subplot illustrates drift rates at apogee, and the right subplot drift rates at perigee. From the figure, it is clear that as eccentricity increases, the formation will drift apart more slowly at apogee, and more quickly at perigee, for a given radius or semi-major axis error.

Figure 4(b) shows similar results, instead assuming no correlation between the two satellites' state estimates. Figure 4(c) shows some additional results, in which a "fudge factor" of $50 \%$ is applied to Eq. (28), and zero correlations assumed. This is intended to capture the type of "poor" velocity estimation that Ref. 3 describes may occur with some GPS-based orbit determination systems.

Finally, it may be of interest to determine the time to drift a given distance due to a semimajor axis error. Since time-to-drift depends inversely on the drift rate, a nonlinear mapping of the statistical moments of the drift rate per orbit is required to find the statistics of the time to drift.a given distance. Let $D$ be the deadband size, i.e. the size of the "control box" in which the satellite must remain, and $\tau$ be the time to reach the deadband. Assume that the relative navigation errors are zero-mean and Gaussian, then due to their linear relationship, the relative drift rate also has a

${ }^{*}$ Ref. 3 shows that one should not always assume that GPS-based orbital navigation systems properly account for this constraint. 
Gaussian probability density,

$$
f(\Delta s)=\frac{1}{\sqrt{2 \pi \sigma_{\Delta s}}} \exp \left(-\frac{\Delta s^{2}}{2 \sigma_{\Delta s}^{2}}\right)
$$

Since

$$
\tau=g(\Delta s)=\frac{D}{\Delta s}
$$

is a nonlinear function, its probability density may be found in terms of Eq. 29 via $^{6}$

$$
f_{y}(y)=\left.\sum_{i=1}^{n} \frac{f_{x}\left(x_{i}\right)}{\left|g^{\prime}\left(x_{i}\right)\right|}\right|_{x_{i}=g^{-1}(y)}
$$

where the $x_{i}$ are the values of $x$ where $y=g(x)$. Using Eqs. (29) and (30) in Eq. (31) results in

$$
f_{\tau}(\tau)=\sqrt{\frac{2}{\pi}} \frac{D}{\sigma_{\Delta s} \tau^{2}} \exp \left(-\frac{D^{2}}{2 \sigma_{\Delta s}^{2} \tau^{2}}\right)
$$

where the fact that the deadband is reached with a positive or negative drift rate has been used.

Figures 5(a) and 5(b) show the probability density and the probability of not reaching the deadband in time $\tau$ for various values of $D / \sigma_{\Delta s}$.

Figure 5(b) can be used for finding the minimum acceptable value of the deadband. For example, if one wants there to be a $75 \%$ probability that the deadband will not be reached in four orbits, then $D / \sigma_{\Delta s}>5$, keeping in mind that this analysis does not include perturbations such as differential drag or thrust errors.

\section{Examples}

Suppose there is some interest in flying a high-eccentricity, $e=0.8$, formation flying mission, in which the satellites are supposed to be $10 \mathrm{~km}$ apart at apogee. If the separation varies by more than $10 \%$ at apogee, or if there is some danger of a collision, the satellites must perform shortduration, relatively high-thrust stationkeeping maneuvers. In order to maximize the science return and mission duration, such maneuvers should not occur more often than every four weeks. The orbit period is approximately one day, so the relative drift at apogee must be less than one kilometer per 28 orbits, or about 36 meters per orbit. Consulting Eq. (27), the relative semi-major axis error should therefore have a "one sigma" value of $36 / \pi=11$ meters. If the relative navigation system is highly correlated, i.e. $\rho_{r v}=-.9$ and $\rho_{i j}=0$, Figure $4(\mathrm{a})$ indicates that a "one sigma" radius error of about 55 meters at apogee should be sufficient to meet the $10 \%$ requirement in a "one sigma" sense. If the navigation system is not well-correlated, i.e. $\rho_{r v}=\rho_{i j}=0$, and the relative velocity errors dominate as with many of the GPS systems described in Ref. 3, then Figure 4(c) 
indicates that a "one sigma" radius error of about 2 meters would be necessary to meet the $10 \%$ requirement.

Note however that at perigee, the 11 meter relative semi-major axis error would produce a drift of about 310 meters per orbit, which may be found using Eq. (5). From Figure 4(a), this drift corresponds to a radius error of $0.6 \mathrm{~m}$ for a well-correlated system. For a poorly correlated system like Figure 4(c) illustrates, the corresponding radius error that would be required at perigee is about $0.15 \mathrm{~m}$. For most elliptical orbit formations, the separation will increase at perigee, so a few kilometer change in relative position may amount to much less than $10 \%$. However, there do exist elliptical orbit formations for which separation at perigee decreases relative to apogee, in which case a collision avoidance maneuver might be required much more often than desired unless the navigation error could be further reduced.

Next, suppose a low-Earth orbiting ( $550 \mathrm{~km}$ altitude), leader-follower mission is proposed in which the separation distance is $60 \mathrm{~m}$, and this must be maintained within $\pm 20 \mathrm{~m}$. The mission will use a highly accurate differential Global Positioning System relative navigator, which can determine relative position to within $6 \mathrm{~cm}$, and relative velocity to within $2 \mathrm{~mm} / \mathrm{sec}$, "one sigma," per axis. From Figure 1, one can see that the corresponding drift uncertainty is about $3 \mathrm{~m}$, that the velocity noise is the limiting error, and that the position/velocity noise combination is such that the value of the correlation coefficient has a reduced effect, since the dominating velocity noise places the position/velocity noise combination above and to the left of the main diagonal of the figure, which is where the curves split based on correlation coefficient (this is consistent with the findings of Ref. 2). It therefore seems appropriate to use the poorly correlated example of Figure 4(c) to assess the drift rate per orbit, from which one can determine the drift to be about $5 \mathrm{~m}$ per orbit. For a $20 \mathrm{~m}$ deadband, one should therefore interpolate between the $D / \sigma_{\Delta s}=3$ and $D / \sigma_{\Delta s}=5$ curves of Figure 5(b), from which it is possible to determine that there is about an $80 \%$ probability that the deadband will not be reached in less than about 5 orbits, or equivalently that there is a $20 \%$ probability that every 5 orbits, the deadband will be reached.

Finally, Figure 6 demonstrates the effect that relative navigation error has on a formation. The figure shows the along-track relative motion time history for one week (105 orbits) for a leaderfollower formation with a desired separation of $100 \mathrm{~m}$ with the $0.3 \mathrm{~mm} / \mathrm{s}$ relative velocity and $6 \mathrm{~cm}$ relative position relative navigation errors described above. The relative state control strategy is a minimum fuel in-track impulsive strategy in which each maneuver consists of three impulses separated by 0.5 orbits. A maneuver is initiated when $75 \%(15 \mathrm{~m})$ of the deadband of $20 \mathrm{~m}$ is reached. $^{7}$ To initiate relative motion there is differential drag, but after the first maneuver the differential drag is set to zero and the only error is the relative navigation error. As Figure 6 shows, when there is a high drift rate due to the semi-major axis error the deadband is exceeded. 


\section{Conclusion}

This paper has presented some guidelines on relative navigation that may be useful for conceptual analysis of formation flying missions. These guidelines generalize previously reported formulae to the case of elliptical orbits. Since the guidelines do not account for non-two-body perturbations, they may be viewed as useful preliminary design tools, rather than as the basis for mission navigation requirements, which should be based on detailed analysis of the mission configuration, including all relevant sources of uncertainty.

\section{Acknowledgment}

The development of this paper benefited from conversations with David Folta and Jon How.

\section{References}

${ }^{1}$ Kirschner, M., Montenbruck, O., and Bettadpur, S., "Flight Dynamics Aspects of the GRACE Formation Flying," 2nd International Workshop on Satellite Constellations and Formation Flying, CNES, Haifa, Israel, February 2001.

${ }^{2}$ How, J. P. and Tillerson, M., "Analysis of the Impact of Sensor Noise on Formation Flying Control," Proceedings of the American Control Conference, 2001, pp. 3986-3991.

${ }^{3}$ Carpenter, J. R. and Schiesser, E. R., "Semimajor Axis Knowledge and GPS Orbit Determination," NAVIGATION: Journal of The Institute of Navigation, Vol. 48, No. 1, Spring 2001, pp. 57-68, also AAS Paper 99-190, Feb., 1999.

${ }^{4}$ Inalhan, G., Tillerson, M., and How, J. P., "Relative Dynamics and Control of Spacecraft Formations in Eccentric Orbits," Journal of Guidance, Control and Dynamics, Vol. 25, No. 1, 2002 , pp. 48-59.

${ }^{5}$ Lear, W. M., "Orbital Elements including the J2 Harmonic," Tech. Rep. 86-FM-18, JSC22213, Rev. 1, Mission Planning and Analysis Divison, NASA Johnson Space Center, Houston, TX, 1987.

${ }^{6}$ Papoulis, A., Probability, Random Variables, and Stochastic Processes, McGraw-Hill, 1984.

${ }^{7}$ Alfriend, K. T. and Lovell, T. A., "Formation Maintenance for Low Earth Near-Circular Orbits," to appear in ASTRODYNAMICS 2003, American Astronautical Society, Univelt, San Diego, CA, 2003. 


\section{List of Table Captions}

Table 1: Speed uncertainty "balancing" radius uncertainty for a range of eccentricities, according to Eq. (28). 


\begin{tabular}{c|ccccc}
\hline & \multicolumn{5}{|c}{$\sigma_{r}[\mathrm{~m}]$} \\
$e$ & 0.01 & 0.18 & 3.1 & 56 & 1000 \\
\hline 0 & $1.2 \mathrm{e}-05$ & 0.00022 & 0.0039 & 0.070 & 1.2 \\
0.35 & $2.4 \mathrm{e}-05$ & 0.00044 & 0.0077 & 0.14 & 2.5 \\
0.7 & $9.4 \mathrm{e}-05$ & 0.0017 & 0.030 & 0.53 & 9.4 \\
0.9 & 0.00074 & 0.013 & 0.23 & 4.2 & 74 \\
\hline
\end{tabular}




\section{List of Figure Captions}

Figure 1: Along-track drift due to semi-major axis error for a typical low earth orbit. ${ }^{3}$ Each family of contours is based on a constant semi-major axis error, resulting from various combinations of radial position error, along-track velocity error, and the correlation between these.

Figure 2: Along-track drift due to semi-major axis differences between two spacecraft in a highly elliptical orbit.

Figure 3: Semi-major axis uncertainty as a function of true anomaly for a particular high Earth orbit example.

Figure 4: Relative drift per orbit due to semi-major axis error for various eccentricities

a): $\rho_{i j}=0.9$.

b): $\rho_{i j}=0$.

c): “Poor" velocity accuracy and zero correlations.

Figure 5: Drift probabilities.

a): Probability density function for time to reach deadband, for various ratios of deadband size to drift rate.

b): Probability of not reaching the deadband in given number of orbits, for various ratios of deadband size to drift rate.

Figure 6: Along-track control error time history for LEO leader-follower formation with semi-major axis errors. 


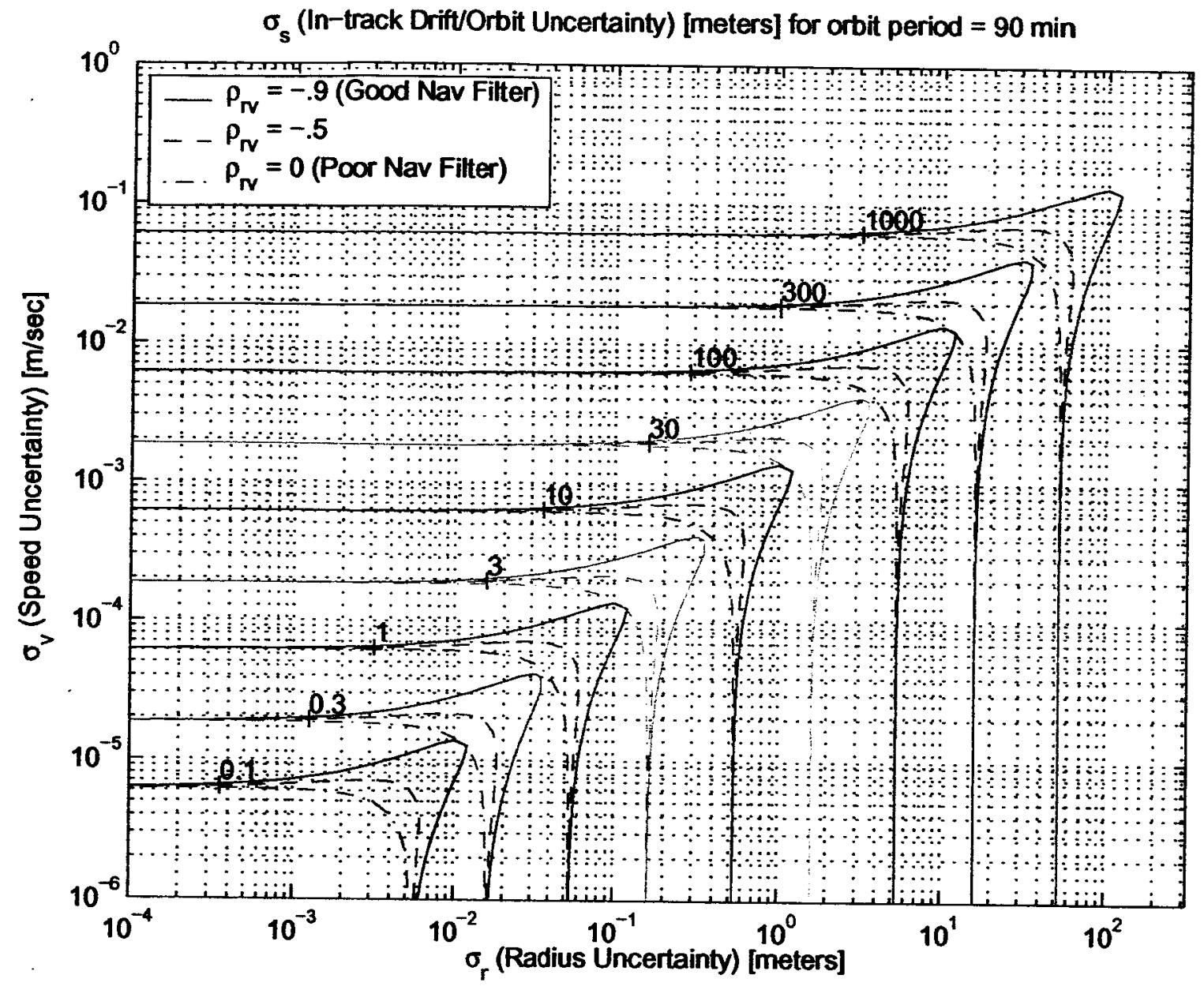




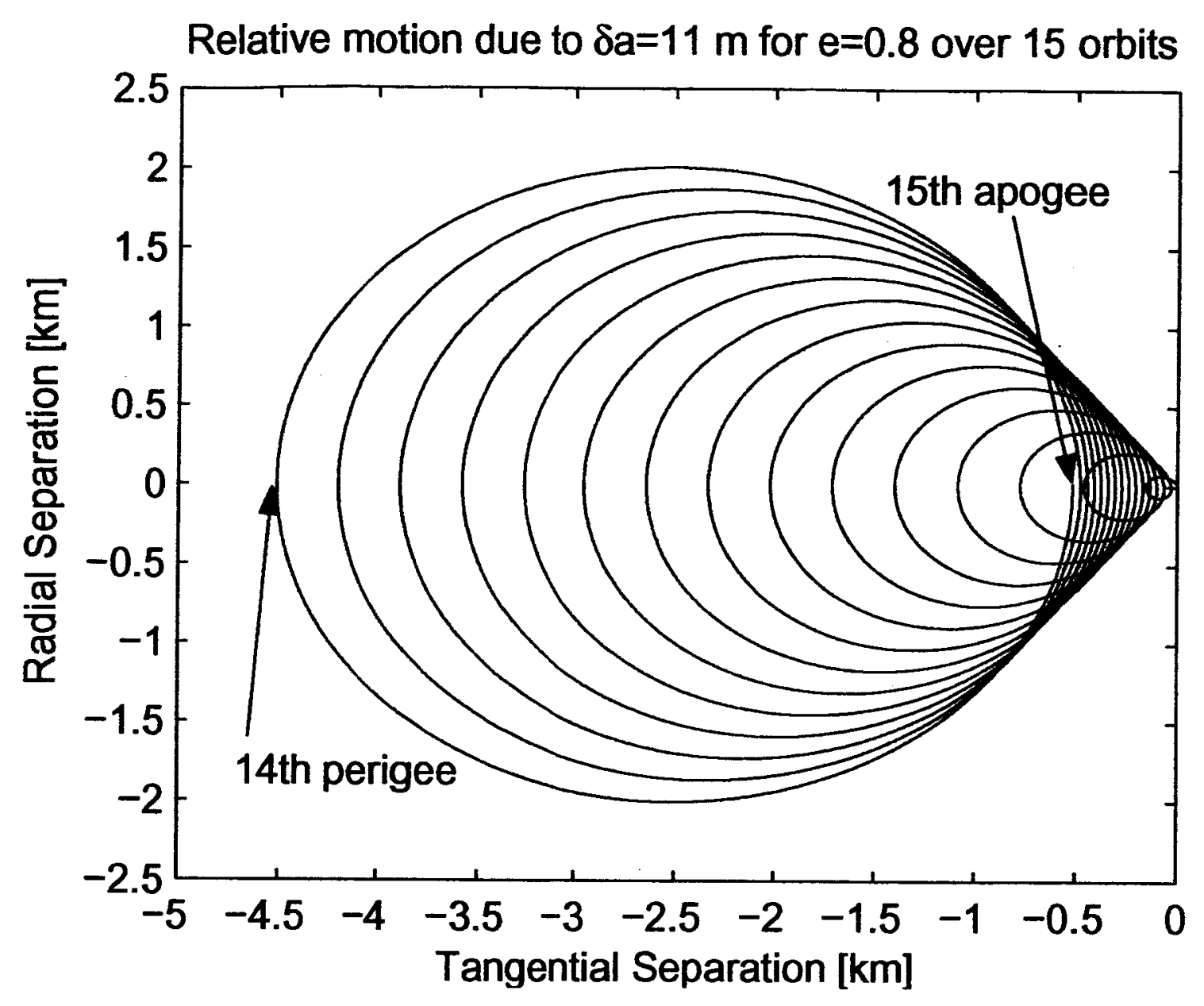




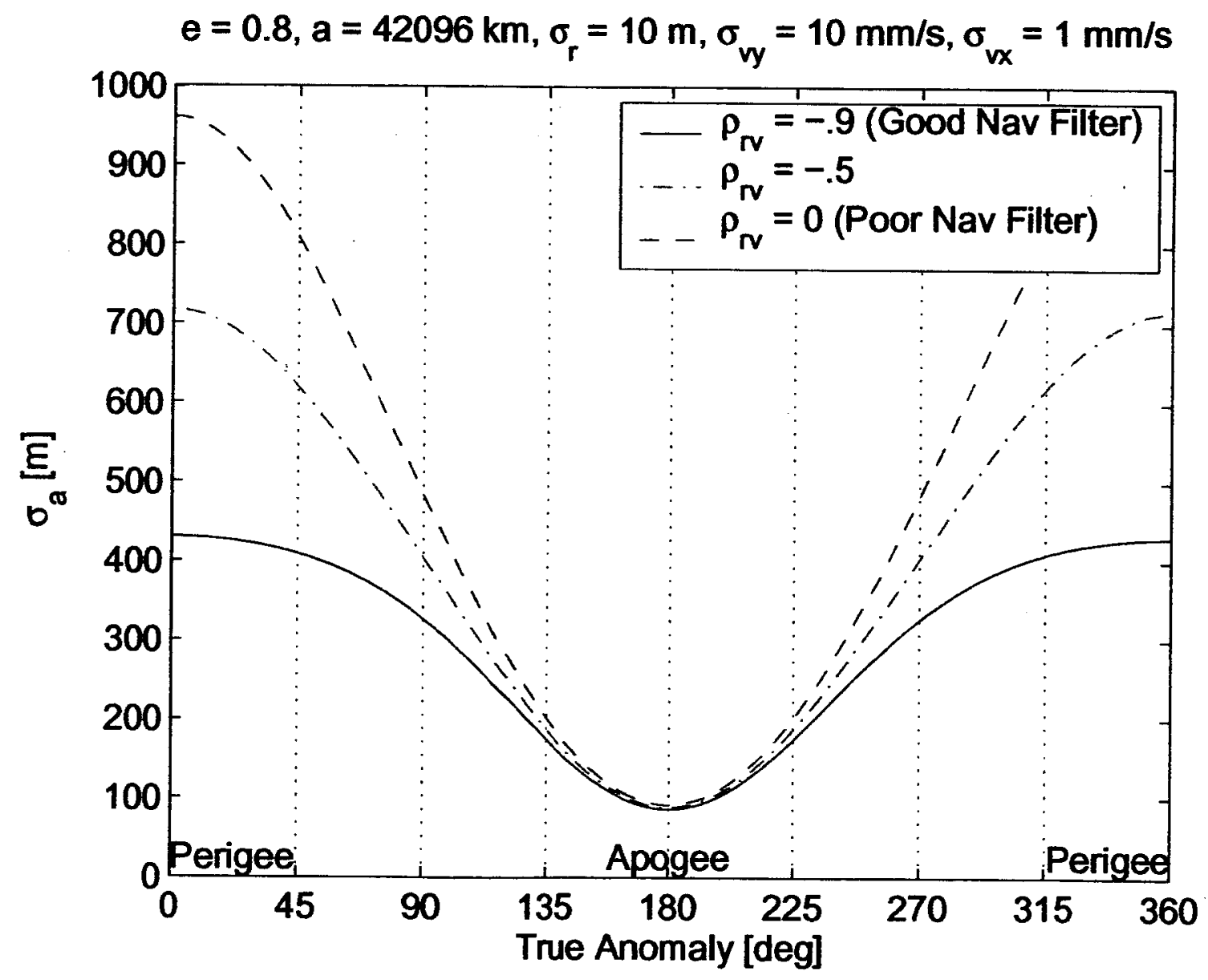



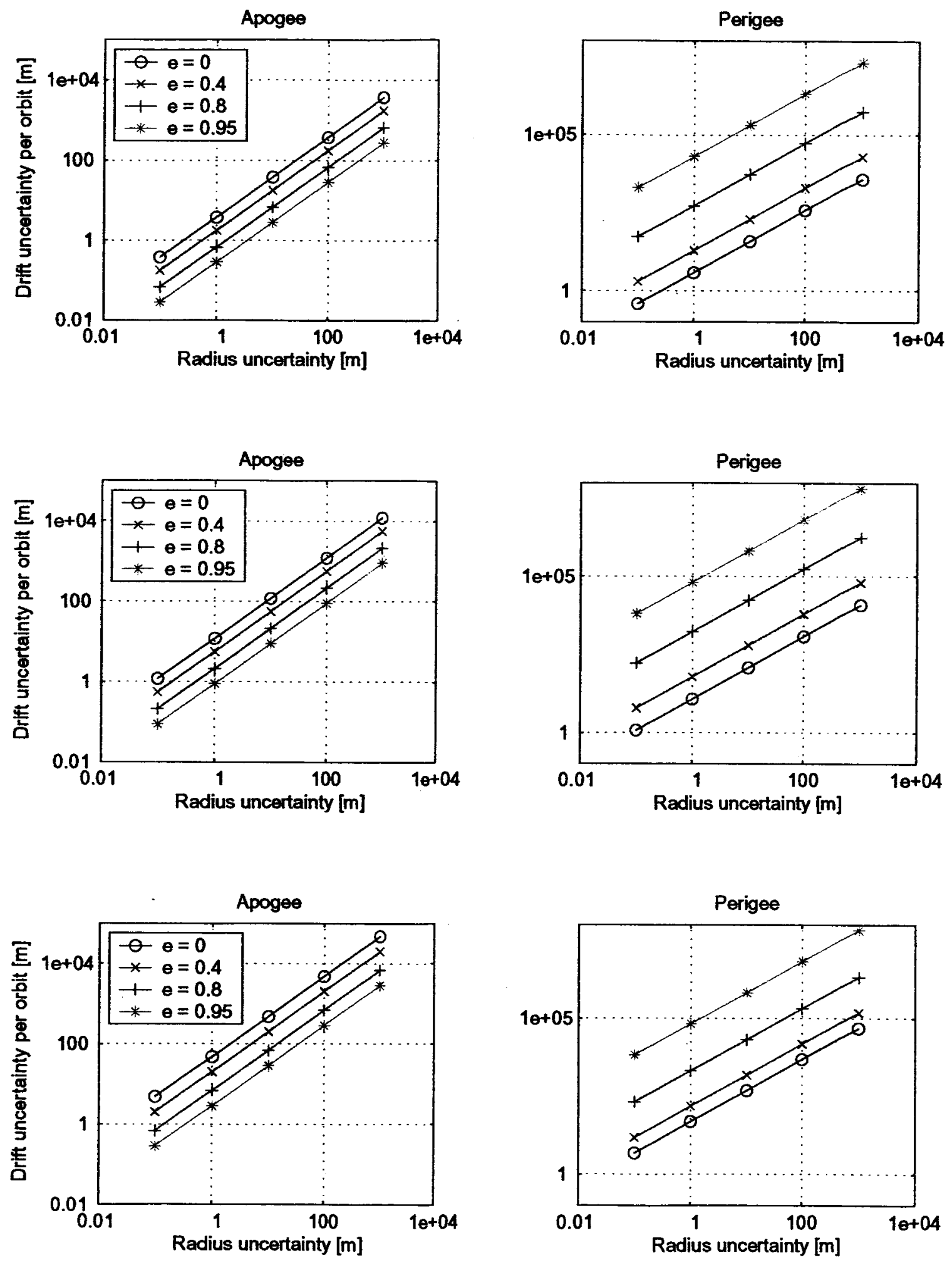

CARPENTER

FIGURE 4:

16 of 18 

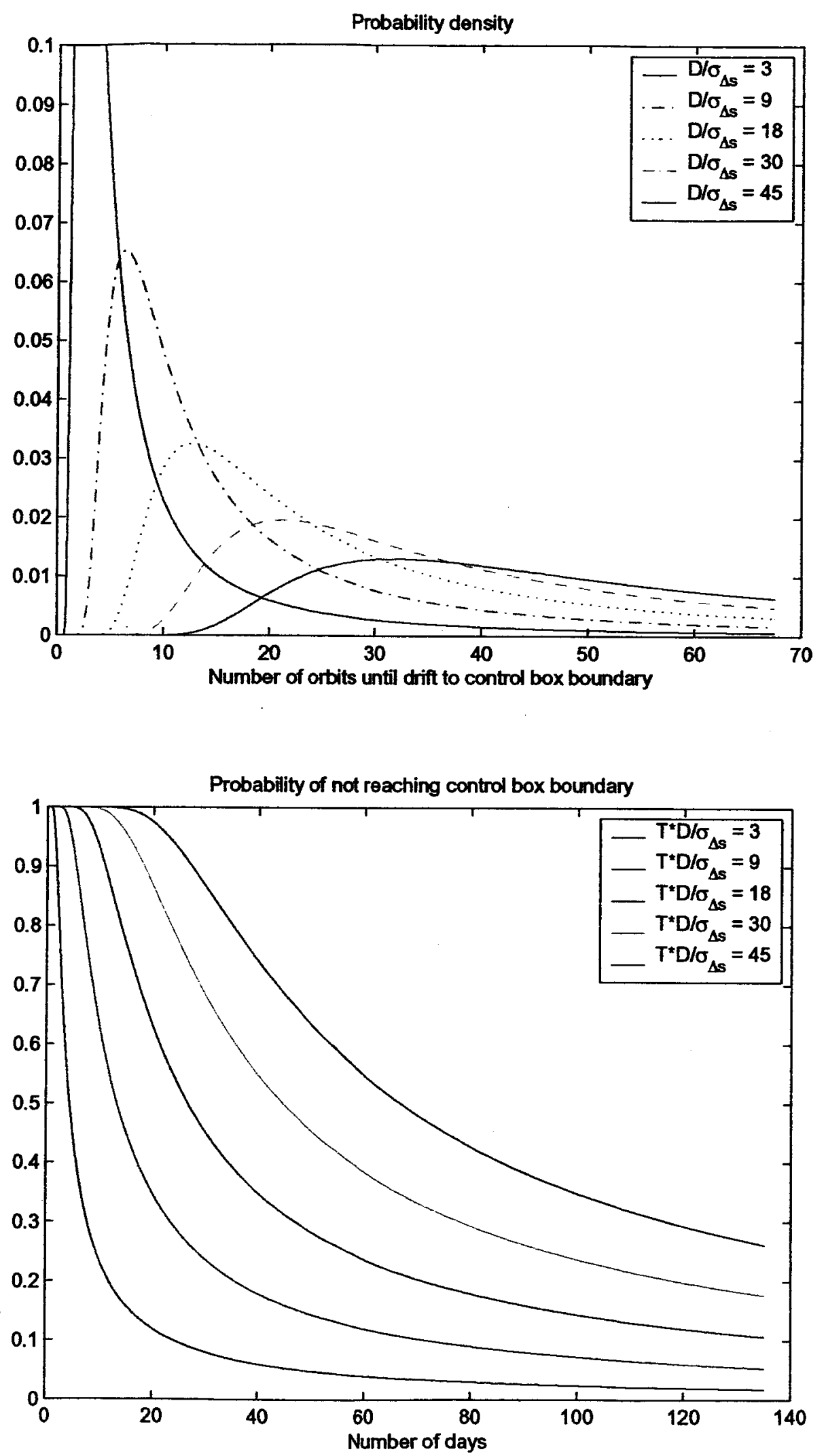

CARPENTER

FIGURE 5: 


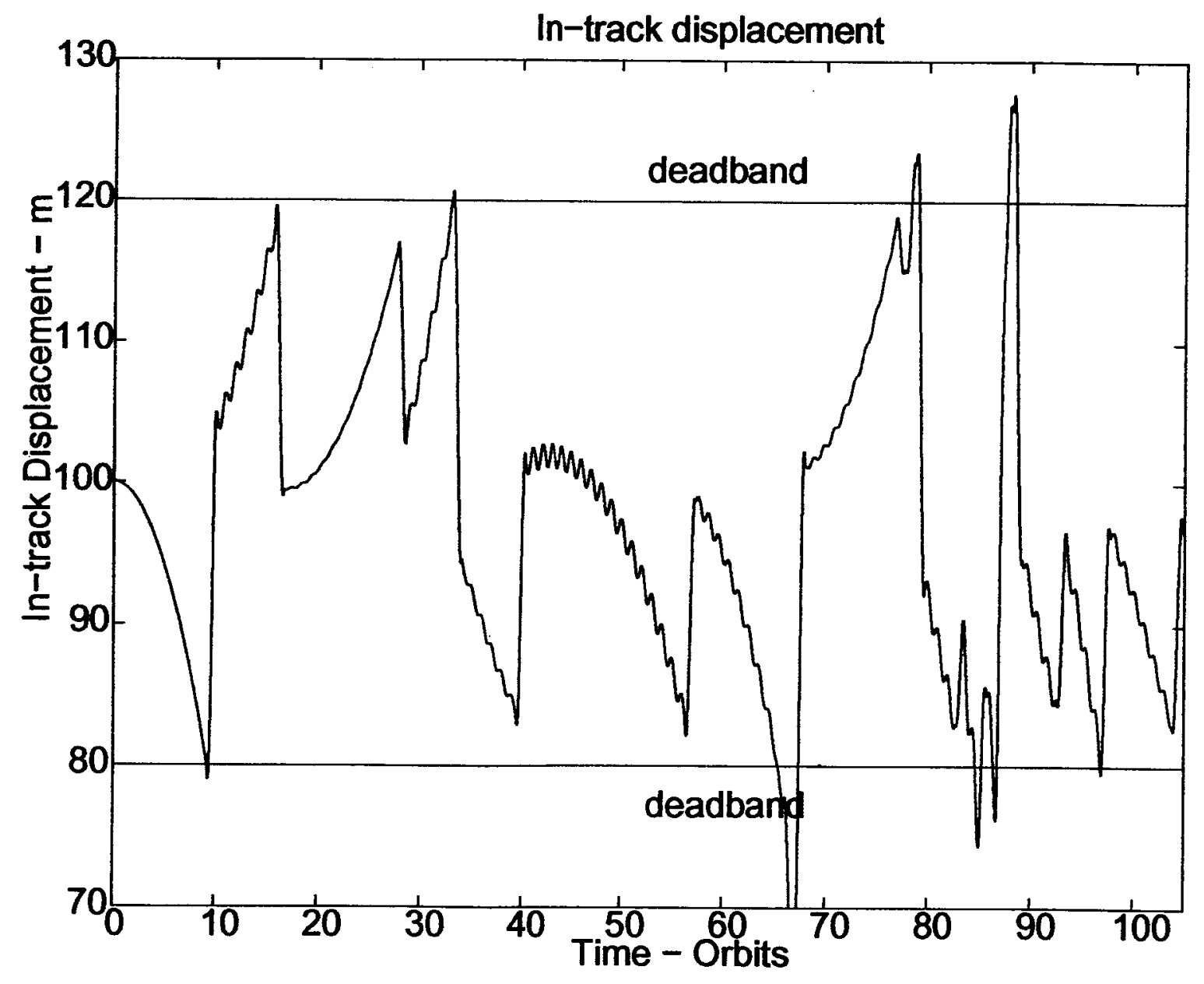

